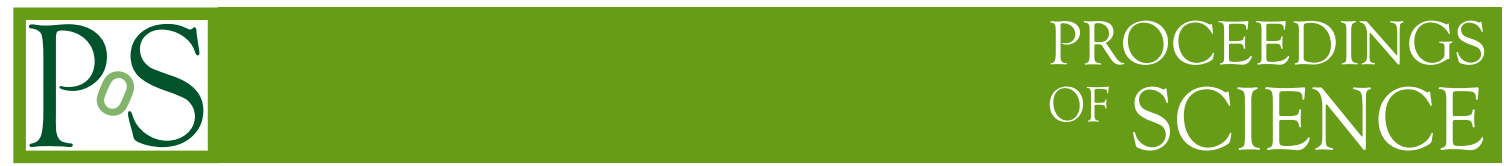

\title{
Progress in Snyder model
}

\section{Salvatore Mignemi*}

Dipartimento di Matematica e Informatica, Universitá di Cagliari, viale Merello 92, 09123

Cagliari, Italy

and INFN, Sezione di Cagliari, Cittadella Universitaria, 09042 Monserrato, Italy

E-mail: smignemi@unica.it

We review the main features of the relativistic Snyder model and its generalizations. We discuss the quantum field theory on this background using the standard formalism of noncommutaive QFT and discuss the possibility of obtaining a finite theory.

Corfu Summer Institute 2019 "School and Workshops on Elementary Particle Physics and Gravity" (CORFU2019)

31 August - 25 September 2019

Corfù, Greece

${ }^{*}$ Speaker. 


\section{Introduction}

Since the origin of quantum field theory (QFT) there have been proposal to add a new scale of length to the theory in order to solve the problems connected to UV divergences. Later, also attempts to build a theory of quantum gravity have proved the necessity of introducing a length scale, that has been identified with the Planck length $L_{p}=\sqrt{\frac{\hbar G}{c^{3}}} \sim 1.6 \cdot 10^{-35} \mathrm{~m}$ [1]. A naive application of this idea, like a lattice field theory, would however break Lorentz invariance. A way to reconcile discreteness of spacetime with Lorentz invariance was proposed by Snyder [2] a long time ago. This was the first example of a noncommutative geometry: the length scale should enter the theory through the commutators of spacetime coordinates.

Noncommutative geometries were however not investigated for a long time, until they revived due to mathematical [3] and physical [4] progresses. Their present understanding is based on the formalism of Hopf algebras [5]. In particular, QFT on noncommutative backgrounds has been largely studied [4]. In most cases, a surprising phenomenon, called UV/IR mixing, occurs: the counterterms needed for the UV regularization diverge for vanishing incoming momenta, inducing an IR divergence. At first, the discover of this property has spoiled the hopes that noncommutative field theory may improve the renormalizability of its commutative counterpart, However, better behaved models were later proposed that avoid this problem [6].

Noncommutative geometries also admit a sort of dual representation on momentum space in theories of doubly special relativity (DSR) [7]. Here a fundamental mass scale is introduced, that causes the curvature of momentum space [8], and the deformation of both the Poincare group and the dispersion relations of the particles. The Snyder model can also be seen as an example of DSR model, where however the Poincaré invariance and the dispersion relations are undeformed.

As mentioned above, Snyder's idea was almost abandoned with the introduction of renormalization techniques, with the exception of some Russian authors in the sixties [9]. It revived more recently, when noncommutative geometry became an important topic of research [10, 11]. However, in spite of several attempts using various methods $[9,12]$, the issue of finiteness of Snyder field theory has not been established up to now. Here we review an attempt to investigate this topic using the formalism of noncommutative QFT [13, 14].

\section{The Snyder model}

The most notable feature of the Snyder model is that, in contrast with most examples of noncommutative geometry, it preserves the full Poincaré invariance. In fact, it is based on the Snyder algebra, a deformation of the Lorentz algebra acting on phase space, generated by positions $x_{\mu}$, momenta $p_{\mu}$ and Lorentz generators $J_{\mu v}$, that obey the Poincaré commutation relations

$$
\begin{gathered}
{\left[J_{\mu \nu}, J_{\rho \sigma}\right]=i\left(\eta_{\mu \rho} J_{v \sigma}-\eta_{\mu \sigma} J_{v \rho}+\eta_{v \rho} J_{\mu \sigma}-\eta_{v \sigma} J_{\mu \rho}\right),} \\
{\left[p_{\mu}, p_{v}\right]=0, \quad\left[J_{\mu v}, p_{\lambda}\right]=i\left(\eta_{\mu \lambda} p_{v}-\eta_{\lambda v} p_{\mu}\right),}
\end{gathered}
$$

together with the standard Lorentz action on position

$$
\left[J_{\mu v}, x_{\lambda}\right]=i\left(\eta_{\mu \lambda} x_{v}-\eta_{v \lambda} x_{\mu}\right)
$$


and a deformation of the Heisenberg algebra, preserving the Jacobi identities,

$$
\left[x_{\mu}, x_{v}\right]=i \beta J_{\mu v}, \quad\left[x_{\mu}, p_{v}\right]=i\left(\eta_{\mu v}+\beta p_{\mu} p_{v}\right)
$$

where $\beta$ is a parameter of the order of the square of the Planck length and $\eta_{\mu \nu}=\operatorname{diag}(-1,1,1,1)$. The generators $J_{\mu \nu}$ can be realized in the usual way as $J_{\mu \nu}=x_{\mu} p_{v}-x_{v} p_{\mu}$.

In contrast with most models of noncommutative geometry, the commutators (2.4) are functions of the phase space variables: this allows them to be compatible with a linear action of the Lorentz symmetry on phase space. However, translations act in a nontrivial way on position variables.

It is important to remark that, depending on the sign of the coupling constant $\beta$, two rather different models can arise:

$$
\begin{array}{ll}
\beta>0 & \text { Snyder model } \\
\beta<0 & \text { anti }- \text { Snyder model }
\end{array}
$$

They have very different properties. For example, the Snyder model has a discrete spatial structure and a continuous time spectrum, while the opposite holds for anti-Snyder.

The subalgebra generated by $J_{\mu \nu}$ and $x_{\mu}$ is isomorphic to the de Sitter/anti-de Sitter algebra, and hence the Snyder/anti-Snyder momentum spaces have the same geometry as de Sitter/antide Sitter spacetime respectively. In fact, the Snyder momentum space can be represented as a hyperboloid $\mathscr{H}$ of equation

$$
\zeta_{A}^{2}=1 / \beta
$$

embedded in a 5D space of coordinates $\zeta_{A}$ with signature $(-,+,+,+,+)$, or equivalently as a coset space $S O(1,4) / S O(1,3)[15]$.

The Snyder commutation relations are recovered through the choice of isotropic (Beltrami) coordinates on $\mathscr{H}$

$$
p_{\mu}=\frac{\zeta_{\mu}}{\sqrt{\beta} \zeta_{4}}
$$

and the identification

$$
x_{\mu}=\sqrt{\beta} M_{\mu 4}, \quad J_{\mu v}=M_{\mu v} .
$$

where $M_{A B}$ are the Lorentz generators in 5D. Note that this construction implies that $p^{2}<1 / \beta$, and hence the existence of a maximal mass, of the order of the Planck mass, for elementary particles. This is a common feature in models with curved momentum space [8].

The momentum space of the anti-Snyder model can be represented analogously, as a hyperboloid of equation (2.5) with $\beta<0$, embedded in a 5D space of coordinates $\zeta_{A}$ with signature $(+,-,-,-,+)$, or equivalently as a coset space $S O(2,3) / S O(1,3)$. Again, anti-Snyder commutation relations are recovered through the choice of isotropic coordinates (2.6) and the identification (2.7). An important difference from the previous case is that the momentum squared is now unbounded. In the following we shall concentrate on Snyder space, but most results hold also for $\beta<0$. 


\section{Generalizations of the Snyder model}

The Snyder model can be generalized by choosing different isotropic parametrizations of the momentum space, but maintaining the identification $x_{\mu}=\sqrt{\beta} M_{\mu 4}$. In this way, eqs. (2.1-2.3) and the position commutation relations still hold, but $\left[x_{\mu}, p_{v}\right]$ is deformed. For example, choosing $p_{\mu}=\zeta_{\mu}$, one obtains [11]

$$
\left[x_{\mu}, x_{v}\right]=i \beta J_{\mu v}, \quad\left[x_{\mu}, p_{v}\right]=i \sqrt{1+\beta p^{2}} \eta_{\mu v} .
$$

The most general choice that preserves the Poincaré invariance is [16]

$$
p_{\mu}=f\left(\zeta^{2}\right) \zeta_{\mu}, \quad x_{\mu}=g\left(\zeta^{2}\right) M_{\mu 4}
$$

Algebraically, the same models can also be obtained by deforming the Heisenberg algebra as ${ }^{1}$ $[11,17]$

$$
\begin{aligned}
& {\left[x_{\mu}, x_{v}\right]=i \beta J_{\mu \nu} \psi\left(\beta p^{2}\right)} \\
& {\left[x_{\mu}, p_{v}\right]=i\left[\eta_{\mu \nu} \phi_{1}\left(\beta p^{2}\right)+\beta p_{\mu} p_{v} \phi_{2}\left(\beta p^{2}\right)\right]}
\end{aligned}
$$

The function $\phi_{1}$ and $\phi_{2}$ are arbitrary, but the Jacobi identity implies

$$
\psi=\phi_{1} \phi_{2}-2\left(\phi_{1}+\beta p^{2} \phi_{2}\right) \frac{d \phi_{1}}{d\left(\beta p^{2}\right)} .
$$

A different kind of generalization is obtained by choosing a curved spacetime (de Sitter) background, imposing nontrivial commutation relations between the momentum variables,

$$
\left[p_{\mu}, p_{v}\right]=i \alpha J_{\mu v}
$$

with $\alpha$ proportional to the cosmological constant. This idea goes back to Yang [19], but was later elaborated in a more compelling way in [20] and further investigated by several authors [21, 22, 23]. We call this generalization Snyder-de Sitter (SdS) model. The other commutation relations are unchanged, except that now, by the Jacobi identities,

$$
\left[x_{\mu}, p_{v}\right]=i\left(\eta_{\mu v}+\alpha x_{\mu} x_{v}+\beta p_{\mu} p_{v}+\sqrt{\alpha \beta}\left(x_{\mu} p_{v}+p_{\mu} x_{v}\right)\right) .
$$

This model depends on two invariant scales besides the speed of light, that are usually identified with the Planck mass and the cosmological constant, from which the alternative name name triply special relativity, proposed in [20] for this model. It must be noted that, in order to have real structure constants, both $\alpha$ and $\beta$ must have the same sign. There are indications that the introduction of $\alpha$ might be necessary in order to obtain a well-behaved low-energy limit of quantum gravity theories [20].

An interesting property of the $\mathrm{SdS}$ model is its duality for the exchange $\alpha x \leftrightarrow \beta p$ [22], that realizes the Born reciprocity [24]. The phase space can be embedded in a 6D space as a coset $\frac{S O(1,5)}{S O(1,3) \times O(2)}$ if $\alpha, \beta>0$, or $\frac{S O(2,4)}{S O(1,3) \times O(2)}$ if $\alpha, \beta<0[23]$.

Alternatively, one can construct the SdS algebra directly from that of Snyder by the nonunitary transformation

$$
x_{\mu}=\hat{x}_{\mu}+\lambda \frac{\beta}{\alpha} \hat{p}_{\mu}, \quad p_{\mu}=(1-\lambda) \hat{p}_{\mu}-\frac{\alpha}{\beta} \hat{x}_{\mu},
$$

where $\hat{x}_{\mu}, \hat{p}_{\mu}$ are generators of the Snyder algebra and $\lambda$ a free parameter [23].

\footnotetext{
${ }^{1} \mathrm{~A}$ less general extension of the model had previously been proposed in [18].
} 


\section{Phenomenological implications}

A wide literature investigates the phenomenological implications of the Snyder model, especially in connection with the generalized uncertainty principle (GUP) [25]. In fact, the deformed Heisenberg algebra of the Snyder model gives rise to a particular case of GUP.

Most papers however consider a nonrelativistic version of the model, where only spatial coordinates present a Snyder structure, while time is unaffected. This case is of course easier to treat and is expected to correspond to the correct low-energy limit (see however [26]). In this context, some standard problems have been discussed, both in classical and quantum settings. Among them, free particle [27, 28], harmonic oscillator [29, 30, 27], hydrogen atom [31], Dirac equation [32], Newtonian orbits [29, 33]. In all these cases, corrections of order $\beta m^{2}$ arise in the equations of motion or in the quantum spectra, with $m$ the mass of the system. The correction are therefore extremely small, except in the case of macroscopic systems, where however it is unlikely that Snyder mechanics applies.

In any case, here we are more interested in the relativistic case, which has obtained much less consideration. Some of the phenomenological consequences are:

- The relativistic uncertainty relations are deformed: from the deformed Heisenberg algebra, for vanishing expectation values of $x$ and $p$, one gets

$$
\Delta x_{\mu} \Delta p_{v} \geq \frac{1}{2}\left(\eta_{\mu v}+\beta \Delta p_{\mu} \Delta p_{v}\right) .
$$

The spatial components of this uncertainty relation essentially coincide with those considered in GUP.

- Modification of perihelion shift of planetary orbits [34]: on a Schwarzschild background the perihelion shift gets an additional contribution to the relativistic one $\delta \theta_{\text {rel }}$,

$$
\delta \theta=\delta \theta_{\text {rel }}\left(1+\frac{5}{3} \beta m^{2}\right),
$$

where $m$ is the mass of the planet. This correction clearly breaks the equivalence principle at Planck scales. It is however not realistic to expect that this formula can be applied to macroscopic objects like planets [35].

- DSR-like effects [36]: no effects of time delay of cosmological photons occur, contrary to other models of noncommutative geometry [37], but some higher-order effects are still present.

To sum up, due to the smallness or absence of observable effects compared to other DSR theories, it seems difficult that verifiable phenomenological predictions can be devised. The reason is that the absence of a deformation of the Poincaré symmetry suppresses the size of the corrections to the standard physical predictions.

\section{Hopf algebras}

In the study of noncommutative models an important tool is given by the Hopf algebra formalism [5], especially in relation with QFT. We recall that in essence an Hopf algebras is an extension of an ordinary algebras in which a structure called coalgebra is added, that describes nontrivial features of tensor products of elements of the algebra by means of an operation called coproduct. 
In particular, since in noncommutative geometry spacetime coordinates are noncommuting operators, the composition of two plane waves $e^{i p \cdot x}$ and $e^{i q \cdot x}$ gives rise to a nontrivial addition rule for the momenta, denoted by $p \oplus q$, that can be described by the coproduct structure of a Hopf algebra, $\Delta(p, q)$. The coproduct is in general noncommutative.

Moreover, the opposite of the momentum is encoded in another operation of the Hopf algebra, called antipode $S(p)$, such that $p \oplus S(p)=S(p) \oplus p=0$.

In the case of the Snyder model, the algebra generated by the $x_{\mu}$ does not close, in fact from (2.4) it is evident that in order to get a closed algebra one should add to the $x_{\mu}$ also the $J_{\mu \nu}$ as primary generators. Although this can be done [15, 38], it is still possible to compute the coproduct and the antipode in the usual way. Of course the result will not obey all the axioms of a Hopf algebra. In particular, the star product will turn out to be nonassociative $[15,11]$.

Geometrically, the nontrivial structure of the addition of momenta can be understood recalling that the momentum manifold of the Snyder model can be represented as a coset space. Calculating the action of the group multiplication on it one can obtain the addition rules of momenta [15].

Alternatively, one can use the algebraic formalism of realizations [11]: a realization of the noncommutative coordinates $x_{\mu}$ is defined in terms of coordinates $\xi_{\mu}, p_{\mu}$ that satisfy canonical commutation relations

$$
\left[\xi_{\mu}, \xi_{v}\right]=\left[p_{\mu}, p_{v}\right]=0, \quad\left[\xi_{\mu}, p_{v}\right]=\eta_{\mu v}
$$

by assigning a function $x_{\mu}\left(\xi_{\mu}, p_{\mu}\right)$ that satisfies the Snyder commutation relations.

The $\xi_{\mu}$ and $p_{\mu}$ are interpreted as operators acting on function of $\xi_{\mu}$, as

$$
\xi_{\mu} \triangleright f(\xi)=\xi_{\mu} f(\xi), \quad p_{\mu} \triangleright f(\xi)=-i \partial f(\xi) / \partial \xi_{\mu}
$$

In particular, it is easy to show that the most general realization of the Snyder model is given by [17]

$$
x_{\mu}=\xi_{\mu}+\beta \xi \cdot p p_{\mu}+\beta \chi\left(\beta p^{2}\right) p_{\mu},
$$

where the function $\chi\left(\beta p^{2}\right)$ is arbitrary and does not affect the commutation relations, but takes into account ambiguities arising from operator ordering of $\xi_{\mu}$ and $p_{\mu}$.

In general, it can be shown [39] that for any noncommutative model,

$$
e^{i k \cdot x} \triangleright e^{i q \cdot \xi}=e^{i \mathscr{P}(k, q) \cdot \xi+i \mathscr{Q}(k, q)},
$$

where the functions $\mathscr{P}_{\mu}$ and $\mathscr{Q}$ can be deduced from the realization. In particular,

$$
e^{i k \cdot x} \triangleright 1=e^{i \mathscr{K}(k) \cdot \xi+i \mathscr{J}(k)},
$$

with $\mathscr{K}_{\mu}(k) \equiv \mathscr{P}_{\mu}(k, 0)$ and $\mathscr{J}(k) \equiv \mathscr{Q}(k, 0)$. The generalized addition of momenta is then given by

$$
k_{\mu} \oplus q_{\mu}=\mathscr{D}_{\mu}(k, q),
$$

with $\mathscr{D}_{\mu}(k, q)=\mathscr{P}_{\mu}\left(\mathscr{K}^{-1}(k), q\right)$, and the coproduct is simply

$$
\Delta p_{\mu}=\mathscr{D}_{\mu}(p \otimes 1,1 \otimes p)
$$


Note that $\mathscr{D}_{\mu}$ is independent of $\chi$. Moreover, the antipode $S\left(p_{\mu}\right)$, is $-p_{\mu}$ for all (generalized) Snyder models. As discussed above, a fundamental property of the Snyder coproduct is its nonassociativity.

For the calculations, it is useful to define a star product, that gives a representation of the product of functions of the noncommutative coordinates $x$ in terms of a deformation of the product of functions of the commuting coordinates $\xi$. In particular, from the previous results one can calculate the star product of two plane waves:

$$
e^{i k \cdot \xi} \star e^{i q \cdot \xi}=e^{i \mathscr{D}(k, q) \cdot \xi+i \mathscr{G}(k, q)},
$$

where

$$
\mathscr{G}(k, q)=\mathscr{Q}\left(\mathscr{K}^{-1}(k), q\right)-\mathscr{Q}\left(\mathscr{K}^{-1}(k), 0\right) .
$$

We consider now a Hermitean realization of the Snyder commutation relations

$$
x^{\mu}=\xi^{\mu}+\frac{\beta}{2}\left(\xi \cdot p p^{\mu}+p^{\mu} p \cdot \xi\right)=\xi^{\mu}+\beta \xi \cdot p p^{\mu}-\frac{5 i}{2} \beta p^{\mu} .
$$

The request of Hermiticity of the operator $x_{\mu}$ will be important for the discussion of field theory.

Applying the previous formalism, one gets after some lengthy calculations [17]

$$
\mathscr{D}_{\mu}(k, q)=\frac{1}{1-\beta k \cdot q}\left[\left(1+\frac{\beta k \cdot q}{1+\sqrt{1+\beta p^{2}}}\right) k_{\mu}+\sqrt{1+\beta p^{2}} q_{\mu}\right],
$$

and

$$
\mathscr{G}(k, q)=\frac{5 i}{2} \ln (1-\beta k \cdot q) .
$$

It follows that

$$
e^{i k \cdot \xi} \star e^{i q \cdot \xi}=\frac{e^{i \mathscr{D}(k, q) \cdot \xi}}{(1-\beta k \cdot q)^{5 / 2}} .
$$

The denominator can be considered as a natural measure related to the curvature of momentum space.

\section{QFT in Snyder space: free fields}

Let us consider a QFT for a scalar field $\phi$ on a Snyder space. Usually, field theories in noncommutative spaces are constructed by writing the action in terms of the star product and continuing to Euclidean signature [4].

In fact, the action functional for a free massive real scalar field $\phi(x)$ in $4 \mathrm{D}$ can be defined through the star product as [17]

$$
S_{\text {free }}[\phi]=\frac{1}{2} \int d^{4} \xi\left(\partial_{\mu} \phi(\xi) \star \partial^{\mu} \phi(\xi)+m^{2} \phi(\xi) \star \phi(\xi)\right) .
$$

The star product of two real scalar fields $\phi(\xi)$ and $\psi(\xi)$ can be computed by expanding them in Fourier series,

$$
\phi(\xi)=\int d^{4} k \tilde{\phi}(k) e^{i k \cdot \xi} .
$$


Then, using (5.12),

$$
\begin{aligned}
& \int d^{4} \xi \psi(\xi) \star \phi(\xi)=\int d^{4} \xi \int d^{4} k d^{4} q \tilde{\psi}(k) \tilde{\phi}(q) e^{i k \cdot \xi} \star e^{i q \cdot \xi} \\
& =\int d^{4} k d^{4} q \tilde{\psi}(k) \tilde{\phi}(q) \int d^{4} x \frac{e^{i \mathscr{D}(k, q) \cdot x}}{(1-\beta k \cdot q)^{5 / 2}}=\int d^{4} k d^{4} q \tilde{\psi}(k) \tilde{\phi}(q) \frac{\delta^{(4)}(\mathscr{D}(k, q))}{(1-\beta k \cdot q)^{5 / 2}} .
\end{aligned}
$$

But

$$
\delta^{(4)}(\mathscr{D}(k, q))=\frac{\delta^{(4)}(q+k)}{\left|\operatorname{det}\left(\frac{\partial \mathscr{D}_{\mu}(k, q)}{\partial q_{v}}\right)\right|_{q=-k}}=\left(1+\beta k^{2}\right)^{5 / 2} \delta^{(4)}(q+k) .
$$

The factor $\left(1+\beta k^{2}\right)^{5 / 2}$ cancels with the denominator of (6.3) and then [17],

$$
\int d^{4} \xi \psi(\xi) \star \phi(\xi)=\int d^{4} \xi \psi(\xi) \phi(\xi) .
$$

This is called cyclicity property, and occurs also in other noncommutative models: it implies that the free theory is identical to the commutative one,

$$
S_{\text {free }}[\phi]=\frac{1}{2} \int d^{4} \xi\left(\partial_{\mu} \phi \partial_{\mu} \phi+m^{2} \phi^{2}\right) .
$$

The propagator is therefore the standard one

$$
G(k) \propto \frac{1}{k^{2}+m^{2}} .
$$

Notice that the cyclicity property is a consequence of our choice of a Hermitian representation for the operator $x$, and can be related to the choice of the correct measure in the curved momentum space.

These results can easily be extended to generalized models of the form (3.3) through a perturbative expansion in $\beta$ [17]. It turns out that the cyclicity property is valid also in that case.

\section{QFT in Snyder space: interacting fields}

The interacting theory is much more difficult to investigate. Several problems arise:

- The addition law of momenta is noncommutative and nonassociative, therefore one must define some ordering for the lines entering a vertex and then take an average.

- The conservation law of momentum is deformed at vertices, so loop effects may lead to nonconservation of momentum in a propagator. This effect is common to other noncommutative QFTs [40].

As an illustration, let us consider the simplest case, a $\phi^{4}$ theory [13]

$$
S_{\mathrm{int}}=\lambda \int d^{4} \xi \phi \star(\phi \star(\phi \star \phi)) .
$$

The parentheses are necessary because the star product is nonassociative. Our definition fixes this ambiguity, but other choices are possible, as for example

$$
S_{\mathrm{int}}^{\prime}=\lambda \int d^{4} \xi(\phi \star \phi) \star(\phi \star \phi) .
$$


With the choice (7.1), the 4-point vertex function turns out to be

$$
G^{(0)}\left(p_{1}, p_{2}, p_{3}, p_{4}\right) \propto \lambda \sum_{\sigma \in S_{4}} \delta\left(\mathscr{D}_{4}\left(\sigma\left(p_{1}, p_{2}, p_{3}, p_{4}\right)\right)\right) \times g_{3}\left(\sigma\left(p_{1}, p_{2}, p_{3}, p_{4}\right)\right),
$$

where

$$
\begin{aligned}
& \mathscr{D}_{4}\left(q_{1}, q_{2}, q_{3}, q_{4}\right)=q_{1}+\mathscr{D}\left(q_{2}, \mathscr{D}\left(q_{3}, q_{4}\right)\right), \\
& g_{3}\left(q_{1}, q_{2}, q_{3}, q_{4}\right)=e^{i \mathscr{G}\left(q_{2}, \mathscr{D}\left(q_{3}, q_{4}\right)\right)} e^{i \mathscr{G}\left(q_{3}, q_{4}\right)},
\end{aligned}
$$

and $\sigma$ denotes all possible permutations of the momenta entering the vertex.

With the expressions of the propagator and the vertex one can compute Feynman diagrams.

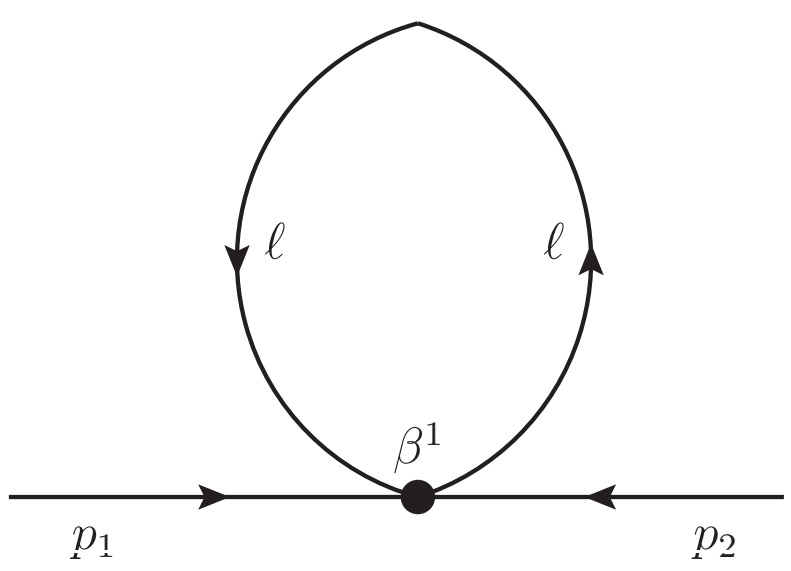

Figure 1: One-loop two-point function.

For example, the one-loop two-point function depicted in fig. 1 in position space is given by

$$
\begin{aligned}
G^{(1)}\left(\xi_{1}, \xi_{2}\right) \propto & \lambda \int d^{4} p_{1} d^{4} p_{2} d^{4} \ell \frac{e^{i p_{1} \xi_{1}}}{p_{1}^{2}+m^{2}} \frac{e^{i p_{2} \xi_{2}}}{p_{2}^{2}+m^{2}} \frac{1}{\ell^{2}+m^{2}} \\
& \times \sum_{\sigma} \delta\left(D_{4}\left(\sigma\left(p_{1}, p_{2}, \ell,-\ell\right)\right)\right) g_{3}\left(\sigma\left(p_{1}, p_{2}, \ell,-\ell\right)\right) .
\end{aligned}
$$

To evaluate the diagram, one must consider the 24 permutations of the momenta entering the vertex. Among these, only 8 conserve the momentum (i.e. $p_{1}=-p_{2}$ ), while the remaining 16 do not.

At the linear level in $\beta$ the calculation can be done explicitly, showing stronger divergences than in the commutative theory. In fact, calculating $n$-points functions, the nonrenormalizability of the linearized theory shows up. The reason is that the improved convergence properties due to the denominator in the star product (5.12) are spoiled if one performs a perturbative expansion in $\beta$.

In return, at first order the results do not depend on the specific ordering chosen for the interaction, and the effects of momentum nonconservation cancel out completely [13].

Attempting instead a calculation to all orders in $\beta$, not all diagrams can be explicitly computed, because it is not possible in general to evaluate explicitly the $\delta$ function in (7.6) [14]. It can be shown, however, that the divergences are suppressed with respect to the noncommutative theory 
and there are even indications that the integrals might be finite, at least for the interaction (7.1). For other interactions, like for example (7.2), it can be shown instead that renormalization is necessary and gives rise to the phenomenon of UV/IR mixing, like in other noncommutative models [4].

We recall however that a model that avoids this problem in the cae of Moyal geometry has been proposed by Grosse and Wulkenhaar [6] (GW model). This model has several attractive properties and gives rise to a perturbatively renormalizable theory to all orders. Its main ingredient are the presence of a duality between position and momentum [41], and the addition to the action of a harmonic oscillator term proportional to $\phi x^{2} \phi$, that smooths the infrared behavior of the theory.

A remarkable observation is that also the Snyder-de Sitter model of sect. 3 enjoys analogous properties [42]. In addition, the harmonic term is induced by the background geometry in a natural way. In fact, using the relation (3.7) between the $\operatorname{SdS}$ and Snyder algebra with $\lambda=0$, and the realization (5.9) of the Snyder algebra, the free action can be reduced, at leading order in $\alpha$ and $\beta$, to

$$
S_{\text {free }}=\int d \xi^{4} \phi\left[p^{2}+\frac{\alpha}{\beta} \xi^{2}+m^{2}+O(\alpha, \beta)\right] \phi,
$$

The free action (7.7) is therefore identical to that of the GW model. Of course, the interaction term is instead different in the two models. Moreover, as discussed in sect. 3, also the SdS model presents a duality between $x$ and $p$.

One may therefore conjecture that also in SdS the IR divergences are suppressed and one can obtain a fully renormalizable theory. The investigation of this topic has been undertaken in ref. [42], but unfortunately in that paper it has only been possible to investigate the first order expansion in $\alpha$ and $\beta$, and the methods used did not permit to explicitly display the duality of the theory. Nevertheless some results concerning the flow of the coupling constants of the linearized theory under the renormalization group have been obtained.

In view of these preliminary results, it would be interesting to pursue the study in this direction, looking for new methods that permit to go beyond the perturbative expansion in the parameter $\alpha$ and $\beta$, to finally settle the question of the consistence of Snyder field theory.

\section{Conclusions}

We have reviewed the present state of research on the Snyder model, the earliest example of noncommutative geometry, and the only one that preserves the Lorentz invariance. Although it seems that no relevant phenomenological predictions can be derived from this model, since the Lorentz invariance suppresses observable effects at low order, the model has a considerable relevance from a theoretical point of view. Also several generalizations can be introduced, notably to the case of a spacetime background of constant curvature.

In particular, the possibility that it may lead to a finite quantum field theory, that was the motive for its introduction, has not yet been ruled out. In this review, we have concentrated on the definition of a QFT using the standard methods of the noncommutative formalism. It turns out that, although an exact calculation has not been performed in full, there is evidence of renormalizability and of the possible absence of UV/IR mixing, at least in the case of a curved background. 


\section{References}

[1] L.J. Garay, Int. J. Mod. Phys. A 10, 145 (1995); S. Hossenfelder, Liv. Rev. Rel. 16, 2 (2013).

[2] H.S. Snyder, Phys. Rev. 71, 38 (1947); Phys. Rev. 72, 68 (1947).

[3] A. Connes, Noncommutative Geometry (Academic Press, 1994); J. Madore, An Introduction to Noncommutative Differential Geometry and its Physical Applications (Cambridge Un. Press, 1999).

[4] For a review, see e.g. M.R. Douglas and N.A. Nekrasov, Rev. Mod. Phys. 73, 977 (2001). R.J. Szabo, Phys. Rept. 378, 207 (2003).

[5] S. Majid, Foundations of quantum group theory (Cambridge University Press, 1995).

[6] H. Grosse and R. Wulkenhaar, Comm. Math. Phys. 256, 305 (2005).

[7] G. Amelino-Camelia, Int. J. Mod. Phys. D 11, 35 (2002); Phys. Lett. B 510, 255 (2001).

[8] J. Kowalski-Glikman, Phys. Lett. B 547, 291 (2002). J. Kowalski-Glikman and S. Nowak, Class. Quantum Grav. 20, 4799 (2003).

[9] Y.A. Gol'fand, JETP 16, 184 (1963); JETP 17, 842 (1963); JETP 37, 356 (1960). V. G. Kadyshevsky, JETP 14, 1340 (1962). R.M. Mir-Kasimov, JETP 22, 629 (1966); JETP 25, 348 (1967).

[10] E. J. Hellund and K. Tanaka Phys. Rev. 94, 192 (1954). G. Jaroszkiewicz, J. Phys. A28, L343 (1995). J.M. Romero and A. Zamora, Phys. Rev.D 70, 105006 (2004); Phys. Lett. B 661, 11 (2008). R. Banerjee, S. Kulkarni and S. Samanta, JHEP 0605, 077 (2006). H.G. Guo, C.G. Huang, Y. Tian, H.T. Wu, Z. Xu and B. Zhou, Class. Quantum Grav. 24, 4009 (2007).

[11] M.V. Battisti and S. Meljanac, Phys. Rev. D 79, 067505 (2009); Phys. Rev. D 82, 024028 (2010).

[12] J.C. Breckenridge, T.G. Steele and V. Elias, Class. Quantum Grav. 12, 637 (1995). T. Konopka, Mod. Phys. Lett. A 23, 319 (2008).

[13] S. Meljanac, S. Mignemi, J. Trampetić and J. You, Phys. Rev. D 96, 045021 (2017). A. FranchinoViñas and S. Mignemi, Phys. Rev. D 98, 065010 (2018).

[14] S. Meljanac, S. Mignemi, J. Trampetić and J. You, Phys. Rev. D 97, 055041 (2018).

[15] F. Girelli and E. Livine, JHEP 1103, 132 (2011).

[16] B. Ivetić and S. Mignemi, Int. J. Mod. Phys. D 27, 1950010 (2018).

[17] S. Meljanac, D. Meljanac, S. Mignemi and R. Štrajn, Phys. Lett. B 768, 321 (2017).

[18] A. Kempff, G. Mangano and R.B. Mann, Phys. Rev. D 52, 1108 (1995). C. Quesne and V.M. Tkachuk, Cz. J. Phys. 56, 1269 (2006).

[19] C.N. Yang, Phys. Rev. 72, 874 (1947).

[20] J. Kowalski-Glikman and L. Smolin, Phys. Rev. D 70, 065020 (2004).

[21] C. Chryssomakolos and E. Okon, Int. J. Mod. Phys. D 13, 1817 (2004). M.C. Carrisi and S. Mignemi, Phys. Rev. D 84, 105031 (2010). R. Banerjee, K. Kumar and D. Roychowdhury, JHEP 1103, 060 (2011). M.M. Stetsko, J. Math. Phys. 56, 012101 (2015).

[22] H.G. Guo, C.G. Huang and H.T. Wu, Phys. Lett. B 663, 270 (2008).

[23] S. Mignemi, Class. Quantum Grav. 26, 245020 (2009).

[24] M. Born, Rev. Mod. Phys. 21, 463 (1949). 
[25] G. Veneziano, Europhys. Lett. 2, 199 (1986). M. Maggiore, Phys. Lett. B 304, 63 (1993).

[26] A. Ballesteros, G. Gubitosi and F. Herranz, arxiv: 1912:12878.

[27] S. Mignemi, Phys. Rev. D 84, 025021 (2011).

[28] Lei Lu and A. Stern, Nucl. Phys. B 854, 894 (2012); Nucl. Phys. B 860, 186 (2012).

[29] S. Benczik, L.N. Chang, D. Minić, N. Okamura, S. Rayyan and T. Takeuchi, Phys. Rev. D 66, 026003 (2002).

[30] C. Leiva, Pramana 74, 169 (2010).

[31] S. Benczik, L.N. Chang, D. Minić, T. Takeuchi, Phys. Rev. A 72, 012104 (2005). M.M. Stetsko, V.M. Tkachuk, Phys. Rev. A 74, 012101 (2006). B. Ivetić, S. Mignemi, and A. Samsarov, Phys. Rev. A 93, 032109 (2016).

[32] C. Quesne and V.M. Tkachuk, J. Phys. A 38, 1747 (2005).

[33] C. Leiva, J. Saavedra and J R Villanueva, Pramana 80, 945 (2013).

[34] S. Mignemi and R. Štrajn, Phys. Rev. D 90, 044019 (2014).

[35] K. P. Gnatenko and V.M. Tkachuk, EPL 126, 50003 (2019).

[36] S. Mignemi and A. Samsarov, Phys. Lett. A 381, 1655 (2017). S. Mignemi and G. Rosati, Class. Quantum Grav. 35, 145006 (2018).

[37] G. Gubitosi and F. Mercati, Class. Quantum Grav. 20, 145002 (2013).

[38] S. Meljanac, S. Mignemi, in preparation.

[39] S. Meljanac, Z. Škoda and D. Svrtan, SIGMA 8, 013 (2012).

[40] G. Amelino-Camelia and M. Arzano, Phys. Rev. D 65, 084044 (2002).

[41] E. Langman and R.J. Szabo, Phys. Lett. B 533, 168 (2002).

[42] A. Franchino-Viñas and S. Mignemi, arxiv:1911.08921. 